\section{OPEN ACCESS}

Edited by:

Zhe $\mathrm{Ma}$,

University of Florida, United States

Reviewed by:

Qiyi Tang,

Howard University, United States

Junji Xing,

Houston Methodist Research

Institute, United States

*Correspondence:

Ruoqing Mao

maoruoging@caas.cn

Yongguang Zhang

zhangyongguang@caas.cn

Yuefeng Sun

sunyuefeng@caas.cn

${ }^{\dagger}$ Present address:

Ruoqing Mao,

Yongguang Zhang,

Yuefeng Sun

Lanzhou Veterinary Research Institute,

Chinese Academy of Agricultural

Sciences, Lanzhou, China

\section{Specialty section}

This article was submitted to

Virology,

a section of the journal

Frontiers in Microbiology

Received: 30 October 2021

Accepted: 06 January 2022

Published: 18 February 2022

Citation:

Hou S, Wang $X$, Ren $S$, Meng $X$ Yin $X$, Zhang J, Tarasiuk K, Pejsak Z,

Jiang T, Mao $R$, Zhang $Y$ and Sun $Y$

(2022) Knockout of HDAC9 Gene

Enhances Foot-and-Mouth Disease

Virus Replication.

Front. Microbiol. 13:805606.

doi: 10.3389/fmicb.2022.805606

\title{
Knockout of HDAC9 Gene Enhances Foot-and-Mouth Disease Virus Replication
}

\begin{abstract}
Shitong Hou' ${ }^{1}$ Xiangwei Wang', Shanhui Ren', Xuelian Meng', Xiangping Yin', Jie Zhang ${ }^{1,2}$, Kazimierz Tarasiuk ${ }^{3}$, Zygmunt Pejsak ${ }^{3}$, Tao Jiang ${ }^{1}$, Ruoqing Mao ${ }^{1 * t}$, Yongguang Zhang ${ }^{1 * t}$ and Yuefeng Sun ${ }^{1 * t}$
\end{abstract}

\begin{abstract}
'State Key Laboratory of Veterinary Etiological Biology, National Foot and Mouth Diseases Reference Laboratory, Key Laboratory of Animal Virology of Ministry of Agriculture, Lanzhou Veterinary Research Institute, Chinese Academy of Agricultural Sciences, Lanzhou, China, ${ }^{2}$ Hebei Normal University of Science and Technology, Qinhuangdao, China,
\end{abstract} ${ }^{3}$ University Center of Veterinary Medicine JU-AU, Krakow, Poland

Foot-and-mouth disease virus (FMDV) is a highly contagious viral disease that mainly infects cloven-hoofed animals. Propagation of FMDV by cell culture is an important method to preserve viral biological and antigenic characteristics, which is crucial in FMD monitoring and vaccine production. However, only a few cell lines are sensitive to FMDV, and there is still a lot of room for improvement. Acetylation is an important posttranslational modification, which is dynamically regulated by histone acetyltransferases (HATs) and histone deacetylases (HDACs). However, the study of the relationship between FMDV and HDACs is still unclear. HDAC9 belongs to the class II of HDACs family; in this study, HDAC9 knockout (KO) BHK-21 cells were successfully established using CRISPR/cas9 technology. The results of karyotype analysis, growth curve analysis, and morphological observation showed that the HDAC9 knockout cell line was stable in growth and morphological characteristics. After infection with FMDV, the expression of viral RNA and protein, viral titers, and the copies of viral RNA in HDAC9-KO cells were significantly higher than those in NC cells. Meanwhile, RNAseq technology was used to sequence HDAC9-KO cells and NC cells infected and uninfected with FMDV. It was found that the differentially expressed innate immune factors containing NFKBIA, SOD2, IL2RG, BCL2L1, CXCL1/2/3, and IL1RAP have significantly enriched in the Jak-STAT, NOD-like receptor, Toll-like receptor, NF-кB, and MAPK signaling pathway. RT-qPCR was performed to detect the expression level of differentially expressed genes and showed consistency with the RNA-seq data. These results preliminarily reveal the role of HDAC9 in host antiviral innate immune response, and the HDAC9-KO cell line could also serve as a useful tool for FMDV research.

Keywords: HDAC9, FMDV, CRISPR/Cas9, RNA-Seq, innate immune response

\section{INTRODUCTION}

Foot-and-mouth disease (FMD) is an acute, highly contagious infectious disease caused by foot-and-mouth disease virus (FMDV), which mainly infects cloven-hoofed livestock and wild animals (Alexandersen and Mowat, 2005). The disease is characterized by vesicular lesions in the buccal cavity, feet, and teats (Grubman and Baxt, 2004), causing severe damages to agricultural development. FMDV belongs to the member of the Aphthovirus genus within the family Picornaviridae. Its genome is a positive single-strand RNA virus with a total length of about $8.4 \mathrm{~kb}$. 
FMDV has seven different serotypes, named O, A, C, Asia 1, SAT1, SAT2, and SAT3. In addition, multiple subtypes were further evolved from each serotype (Knowles and Samuel, 2003). FMD vaccine development faces many challenges because there is no cross-protection between different serotypes (Klein, 2009).

Acetylation is an important post-translational modification, including histone and non-histone acetylation (Glozak et al., 2005). Previous studies have shown that protein acetylation affects the infection and immune process of virus in many ways, such as viral protein acetylation, histone acetylation in the promoter region of host immune-related genes, and acetylation of immune signaling molecules (Kiernan et al., 2003; Nusinzon and Horvath, 2003; Choi et al., 2016; Hatakeyama et al., 2018). The levels of protein acetylation are dynamically regulated by histone acetyltransferase (HAT) and histone deacetylase (HDAC). There are 18 members of the mammalian HDACs family. At present, 18 different HDACs are divided into four categories: Class I is $\mathrm{HDAC1}, 2$, 3, and 8, mainly located in the nucleus; Class II is HDAC4, 5, 6, 7, 9, and 10, which can shuttle between nucleus and cytoplasm; Class III is members of SIRT family, including Sirt 1-7; Class IV is HDAC 11, mainly located in the nucleus (de Ruijter et al., 2003). Different HDACs have specific functions without obvious functional redundancy. Individual members of the HDACs family have been shown to play important regulatory roles in the process of virus-host interactions (Feng et al., 2018). Previous studies have shown that methyltransferase Dnmt3a upregulates HDAC9 to deacetylate the kinase TBK1 for activation of antiviral innate immunity ( $\mathrm{Li}$ et al., 2016). However, the role of protein acetylation and HDACs family genes in FMD infection is unclear.

BHK-21 (baby hamster kidney) cell lines and PK-15 (porcine kidney) cell lines (Zhang et al., 2018) have been successfully used for FMDV research and vaccine development. BHK-21 cells grow rapidly and have a wide sensitive spectrum of viruses. Subsequently, it was used for the proliferation of various viruses and vaccine production, such as FMD vaccine, rabies vaccine, and Newcastle disease vaccine (Amadori et al., 1997).

In this study, HDAC9-KO cell lines were established by using CRISPR/cas9 technology and identified by a series of experiments. Furthermore, the RNA-seq technology was used to reveal the signal transduction pathway of host-virus interaction at the mRNA level during FMDV infection. The differentially expressed genes (DEGs) were analyzed between FMDV-infected and non-infected NC and HDAC9-KO cells. Kyoto Encyclopedia of Genes and Genomes (KEGG) pathways were significantly enriched in the different signaling pathways; the typical genes associated with immune pathways were selected for verification by real-time quantitative PCR (RT-qPCR).

\section{MATERIALS AND METHODS}

\section{Cell and Viruses}

Baby hamster kidney cells (BHK-21 cell) and porcine kidney cells (PK-15 cell) were maintained in Dulbecco's modified Eagle's medium (DMEM) supplemented with $10 \%$ fetal bovine serum
(FBS) and 1\% penicillin-streptomycin in a humidified incubator at $37^{\circ} \mathrm{C}$ with $5 \% \mathrm{CO}_{2}$.

FMDV O/BY/2010 strain is stored in the National FMD Reference Laboratory (Lanzhou, Gansu, P.R. China). FMDV was propagated in BHK-21 cells, and the virus titers were determined by $50 \%$ tissue culture infectious dose $\left(\mathrm{TCID}_{50}\right)$, which was calculated by the Reed-Muench formula. All virusrelated experiments were conducted in the Biosafety Level-3 (BSL-3) Laboratory of Lanzhou Veterinary Research Institute following the standard protocols and biosafety regulations provided by the Institutional Biosafety Committee.

\section{Establishment of HDAC9 Knockout BHK-21 Cell Line}

HDAC9-Knockout (KO) cell lines were established by using the CRISPR/cas9 system following the published protocols (Ran et al., 2013; Haeussler et al., 2016). To establish the HDAC9-KO cell lines, two pairs of the small guide RNAs (sgRNA) targeting the HDAC9 gene of BHK-21 cells were designed using the CRISPOR tool. ${ }^{1}$ Two sgRNAs were annealed and ligated to pspcas9 (BB) plasmid. The molecularly confirmed plasmids were transfected into BHK-21 cells, 0.3 $\mu \mathrm{g}$ puromycin was added per $1 \mathrm{~mL}$ of DMEM supplements, and the medium was renewed every 2 days. Single-cell clone was selected by clone ring anchoring method after puromycin selection. The obtained monoclonal cell lines were identified by sequencing and Western blotting. The primers used for plasmid construction and sequencing are listed in Table 1.

\section{Western Blotting}

Cell precipitate was lysed in RIPA Lysis Buffer containing a $1 \%$ protease inhibitor cocktail. Total protein concentrations were quantified using the BCA Protein Assay kit. Equal amounts of protein samples were separated by sodium dodecyl sulfate-polyacrylamide gel electrophoresis (SDSPAGE) and transferred to polyvinylidene difluoride (PVDF) membrane. Then, the membrane was blocked with 5\% skim milk at room temperature for $1 \mathrm{~h}$, and the membranes were incubated with primary antibody overnight at $4^{\circ} \mathrm{C}$. After that, the membrane was washed with TBST three times before incubation with HRPconjugated secondary antibody for $1 \mathrm{~h}$ followed by chemiluminescent detection.

${ }^{1}$ http://crispor.tefor.net/

TABLE 1 | The primers of HDAC9 used for plasmid construction and sequencing.

\begin{tabular}{ll}
\hline Gene & Sequence $\left(\mathbf{5}^{\prime} \rightarrow \mathbf{3}^{\prime}\right)$ \\
\hline HDAC9-g26-fw & CACCGGTGAAGTCCGAGGTTCCGAT \\
HDAC9-g26-rv & AAACATCGGAACCTCGGACTTCACC \\
HDAC9-g26GT-FP & ACGTTGGTTAAGTGGTCCTGT \\
HDAC9-g26GT-RP & GGTCAGGTTCTCATGCTGCT \\
\hline
\end{tabular}




\section{Virus Infection, RNA Extraction, and RT-qPCR}

BHK-21 cells were cultured in a $60-\mathrm{mm}$ dish to reach an approximate $90 \%$ confluence, which was washed once with PBS and incubated with FMDV at a multiplicity of infection (MOI) of 0.1 at $37^{\circ} \mathrm{C}$ for $1 \mathrm{~h}$. Then, the cells were washed again with PBS and cultured in $3 \mathrm{~mL}$ of FBS-free DMEM. After infection, the supernatant was removed and $1 \mathrm{~mL}$ of TRIzol Reagent (Invitrogen) was added to each dish. Total RNA was isolated according to the manufacturer's instructions. RNA (1 $\mu \mathrm{g})$ was used as the template for cDNA synthesis using PrimeScript ${ }^{\mathrm{TM}}$ RT reagent Kit with gDNA Eraser (TAKARA). cDNA was then subjected to real-time PCR quantification using SYBR green Premix Ex Taq II (TAKARA). $\beta$-Actin gene was used as an internal control. The primers used in the experiment are listed in Table 2. All the experiments were repeated at least three times, and relative mRNA expression levels were calculated using the threshold cycle $\left(2^{-\Delta \Delta C t}\right)$ method.

\section{Growth Characteristics of Foot-and-Mouth Disease Virus in HDAC9 Knockout Cells}

The distribution of FMDV in HDAC9-KO cells was detected by indirect immunofluorescence assay (IFA). NC and HDAC9$\mathrm{KO}$ cells were infected with FMDV at an MOI of 0.1 for $6 \mathrm{~h}$. The cells were washed 3 times and fixed with $4 \%$ paraformaldehyde for $1 \mathrm{~h}$, permeabilized with $0.1 \%$ Triton $\mathrm{X}-100$ for $5 \mathrm{~min}$, and blocked with $3 \%$ BSA for $1 \mathrm{~h}$. The cells were incubated with anti-VP1 antibody (1:500 dilution by $1 \% \mathrm{BSA}$ ) at $4^{\circ} \mathrm{C}$ overnight. Then, the cells were incubated with goat anti-rabbit IgG-FITC antibody (1:500 dilution by $1 \% \mathrm{BSA}$ ) at $37^{\circ} \mathrm{C}$ for $1 \mathrm{~h}$, and the nuclei were stained with DAPI. Finally, the cells were observed by confocal microscopy.

The growth characteristics of FMDV in NC and HDAC9-KO cells were detected by qRT-PCR. HDAC9-KO cells and NC cells were seeded in 24 -well plates $\left(5 \times 10^{5}\right.$ cells/well) for $24 \mathrm{~h}$ and then infected with the FMDV at an MOI of 0.001 when confluence reached 90\%. Viral genome RNAs were extracted using a Viral RNA kit (Omega). The platinum Quantitative RT-PCR kit was used for the qRT-PCR assay, and the primer and probe sequence are shown as the supporting reference (Mao et al., 2018). All the experiments were repeated at least three times.

\section{$\mathrm{TCID}_{50}$ Assay}

Virus titers were determined using $\mathrm{TCID}_{50}$ assay. BHK-21 cells were seeded in 96-well plates with 90\% confluence, and a series of 10 -fold serial dilutions from $10^{-1}$ to $10^{-8}$ of virus samples were prepared in another plate. One hundred microliters of the above samples were added to each well, and the plates were incubated at $37^{\circ} \mathrm{C}$ for $1 \mathrm{~h}$. Then, the inoculum was removed and cells were cultured in DMEM supplemented with 1\% FBS for $72 \mathrm{~h}$. All plates were analyzed by microscopic examination to determine the cytopathogenic effect (CPE). TCID 50 was calculated by the Reed-Muench method (Reed and Muench, 1937).
TABLE 2 | Primers used for RT-qPCR amplification.

\begin{tabular}{|c|c|}
\hline Gene & Sequence $\left(5^{\prime} \rightarrow 3^{\prime}\right)$ \\
\hline VP1-qFP & GACAACACCACCAACCCA \\
\hline VP1-qRP & ССТTCTGAGCCAGCACTT \\
\hline 3D-qFP & ACTGGGTITACAAACCTGTGA \\
\hline 3D-qRP & GCGAGCCCTGCCACGGA \\
\hline$\beta$-Actin-qFP & GCTGGCCGGGACCTGACAGACTACC \\
\hline$\beta$-Actin-qRP & TCTCCAGGGAGGAAGAGGATGCGGC \\
\hline NFKBIA-qFP & ACCAACTACAACGGCCACA \\
\hline NFKBIA-qRP & GGCCCCAGGTAAGCTGATAG \\
\hline BCL2L1-qFP & AATCAGCTCGCAGATCCGAA \\
\hline BCL2L1-qRP & GAGATGGGCTCAACCAGTCC \\
\hline CYLD-qFP & AGGCCTATGGAGCCAAGAGA \\
\hline CYLD-qRP & GCCTITGCGGAAGGAACTC \\
\hline CALM1-qFP & ACGATTGAGCACAGTCAGGC \\
\hline CALM1-qRP & TTCTGGGGCTGTGTCTCCAA \\
\hline IGFBP5-qFP & GTTGTTGGGTGAGGGCAC \\
\hline IGFBP5-qRP & CПTCTCATCGCAGGGCTCA \\
\hline CXCL1/2/3-qFP & CCAGCACCTCAACTCCAGAC \\
\hline CXCL1/2/3-qRP & GGTGAACCCCTGTCATGGTC \\
\hline CCL20-qFP & TCCGTGTGTGCTGATCCAAA \\
\hline CCL20-qRP & GTCTGTGCAATGACGTGCAG \\
\hline SOD2-qFP & GAGCCCTAATGGTGGTGGAG \\
\hline SOD2-qRP & GCAGCAATCTGTAAGCGACC \\
\hline PLEKHA4-qFP & ACCACATCGTCTGTGAGCAG \\
\hline PLEKHA4-qRP & CTGACACTGTAGCTGGGCAA \\
\hline IL2RG-qFP & AATCTTGTGATCCCCTGGGC \\
\hline IL2RG-qRP & ACAGAAGGATTCTAGTTTCTGTCCA \\
\hline BIRC3-qFP & ATTTAAAGGCGTCGTGGCG \\
\hline BIRC3-qRP & TACTAGGCTGAACACCGCAG \\
\hline TRAF2-qFP & TCGAGGGTGCTGCTCTAGTA \\
\hline TRAF2-qRP & GGGAAAGCAAGCCACCAAAG \\
\hline
\end{tabular}

\section{Transcriptome RNA-Seq}

The differential expression of mRNA in FMDV-infected and uninfected HDAC9-KO cells and NC cells was detected to identify the pathways of innate immunity using RNA sequencing (RNA-seq). The cells were infected with FMDV at an MOI of 0.1 for $6 \mathrm{~h}$. The samples were washed three times with PBS, and total RNA was extracted using TRIzol Reagent. These samples were analyzed at the transcriptome level using RNAseq at BGI Genomics (Wuhan, China). The data were obtained by sequencing named raw reads and then quality control (QC) was performed. High-quality reads were aligned to the reference genome. After that, gene quantitative analysis and various analyses based on gene expression level (principal component, correlation, differential gene screening, etc.) were carried out, and the DEGs between samples were analyzed. Fragments per kilobases per million fragments method (FPKM) was used to evaluate the expression level of Unigene and the fold change between different samples. The DEGs were selected with $\log _{2}$ (fold change) $>1$ or $\log _{2}$ (fold change) $<-1$ and statistical significance $(p$-value $<0.05)$.

\section{Karyotype Analysis}

HDAC9-KO cells and NC cells were treated with $0.1 \mu \mathrm{g} / \mathrm{ml}$ colchicine at $37^{\circ} \mathrm{C}$ for $6 \mathrm{~h}$ to obtain metaphase cells, which were 
harvested and the numbers of chromosomes were determined. Then, the G-banding technique is used to identify individual pairs of chromosomes based on size and space.

\section{Cell Proliferation Assay}

Cell proliferation was assayed using Cell-Counting Kit 8 (CCK8) at the indicated time points. HDAC9-KO cells and $\mathrm{NC}$ cells were seeded in 96-well plates $\left(1 \times 10^{4}\right.$ cells/well $)$. To measure the cell viability at the indicated time points, CCK 8 reagent was added to each well for $10 \mu \mathrm{L}$ and incubated for $1 \mathrm{~h}$. After that, the absorbance at $450 \mathrm{~nm}$ was determined with Varioskan ${ }^{\mathrm{TM}}$ LUX multimode microplate reader (Thermo).

\section{Statistical Analysis}

All the experiments were repeated at least three times, the statistical significance was evaluated by Student's $t$-test using the SPSS 17.0 software.

\section{RESULTS}

\section{Successfully Established HDAC9 Knockout BHK-21 Cell Lines}

HDAC9 knockout (HDAC9-KO) BHK-21 cells were established using the CRISPR/cas9 system. Two pairs of the small guide RNAs (sgRNA) were designed by the CRIPOR tool and ligated to the pspcas9 (BB) plasmid. Single-cell clones were picked using the cloning-ring anchoring method and identified after extended culture. The gene of HDAC9 in the cell line was confirmed by DNA sequencing. The results showed that one nucleotide insertion was detected in the first exon of one allele of HDAC9 (HDAC9-KO-1), and a 55-nucleotide insertion was introduced into the other allele of HDAC9 (HDAC9-KO-2) (Figures 1A,B). The expression of HDAC9 in the cell line was confirmed by Western blotting. The results showed that HDAC9 was successfully knocked out in two BHK-21 cell lines compared with the NC cell line (Figure 1C). Then, the cell growth curve was created and the results showed that there was no significant difference in growth rate between HDAC9KO cell lines (HDAC9-KO-1 and HADC9-KO-2) and NC [cells transfected with empty pspcas9 (BB) plasmid and selected by puromycin] (Figure 1D), suggesting that the HDAC9-KO cell lines could be extended indefinitely with steady growth rate. As shown in Figure 1E, HDAC9 was mainly distributed in the nucleus, and more green fluorescence signals were observed in NC cells compared with HDAC9-KO cells.

Both structural and number variations in chromosomes were detected using karyotype tests. Chromosome numbers of HDAC9-KO-1 cells and wild-type BHK-21 cells were analyzed. We found no evidence for an absolute difference between chromosome numbers in HDAC9-KO-1 cells and wild-type BHK-21 cells, which both have 42 chromosomes (Figures 2A,C). The G-banding technique was used to confirm whether the process of gene knockout induced chromosomal structural aberrations of HDAC9-KO-1 cells. As shown in Figures 2B,D, all chromosomes were unchanged in the HDAC9-KO-1 cells, and the cell line had the same karyotype as the wild-type BHK-21 cells without chromosomal aberration.

\section{HDAC9 Knockout Enhances Foot-and-Mouth Disease Virus Replication}

NC and HDAC9-KO-1 cells were infected with FMDV at an MOI of 0.1 . The cytopathic effect (CPE) could be observed at $4 \mathrm{~h}$ after virus infection, and more $\mathrm{CPE}$ was observed in HDAC9-KO-1 cells (Figure 3A). Indirect immunofluorescence experiments were carried out to further visualize the distribution of FMDV in NC and HDAC9-KO-1 cells. As shown in Figure 3B, viral structure protein VP1 was mainly distributed in the cytoplasm and more green fluorescence signals were observed in HDAC9-KO-1 cells compared with NC cells.

TSA (trichostatin A) and SAHA (vorinostat) are broadspectrum HDAC inhibitors, and NAM is an inhibitor of SIRT family. As shown in Figure 4A, the expression of VP1 protein increased significantly after TSA and SAHA treatment. This suggests that HDACs rather than SIRT families can regulate FMDV replication. Equal amounts of FMDV $(\mathrm{MOI}=0.1)$ were incubated with NC and HDAC9-KO-1 cells; the viral mRNA levels of structural protein VP1 and non-structural protein $3 \mathrm{D}$, and the expression of VP1 protein and viral titers were determined and compared. As shown in Figures $4 \mathbf{B}-\mathbf{D}$, the expression of viral RNA and protein, as well as the viral titers, was significantly higher in HDAC9-KO-1 cells compared to the NC cells. Then, total viral RNA of FMDV $(\mathrm{MOI}=0.001)$ was collected, and the viral growth curve was detected by qRT-PCR. As shown in Figure 4E, the number of RNA copies of the FMDV increased acutely from $6 \mathrm{~h}$ post-infection, and the growth rate is the fastest in $12-36 \mathrm{~h}$. It was also found that compared with NC cells, the HDAC9-KO-1 cells had a higher number of viral copies and a faster viral replication rate. These results showed that knockout of HDAC9 gene enhances FMDV replication in the BHK-21 cell line.

To further confirm the above results, RNA interference technique was performed in PK-15 cells. The knockdown effects were confirmed by Western blot analysis (Figure 5A). As shown in Figures 5A-C, compared with the control siRNA transfected cells (NC), the viral protein level, VP1 and 3D mRNA levels were significantly increased. These results indicated that the knockdown of HDAC9 by siRNA significantly enhanced FMDV replication in PK-15 cells.

\section{Foot-and-Mouth Disease Virus Triggered a Lesser Degree Anti-Virus Innate Immune Response in HDAC9-KO Cells}

RNA-seq technology was used to sequence the gene expression of NC and HDAC9-KO-1 cells infected with FMDV or not. The relevant clean data have been uploaded to the NCBI database. ${ }^{2}$ The sequencing generated a

\footnotetext{
${ }^{2}$ https://www.ncbi.nlm.nih.gov/sra/PRJNA777823
} 
A

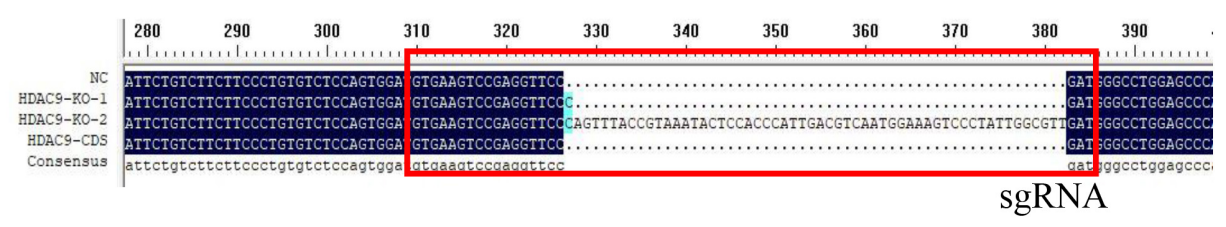

B

NC

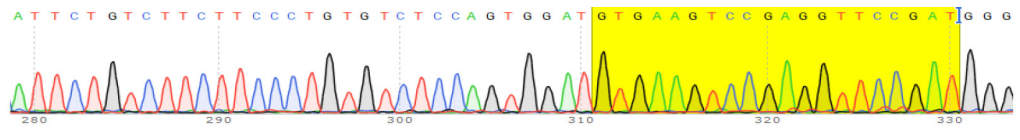

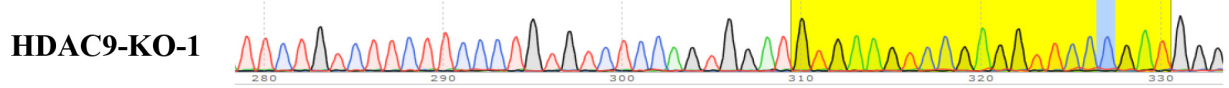

HDAC9-KO-2

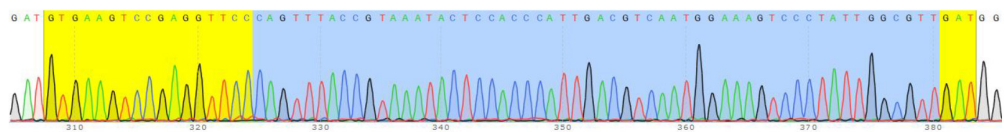

C
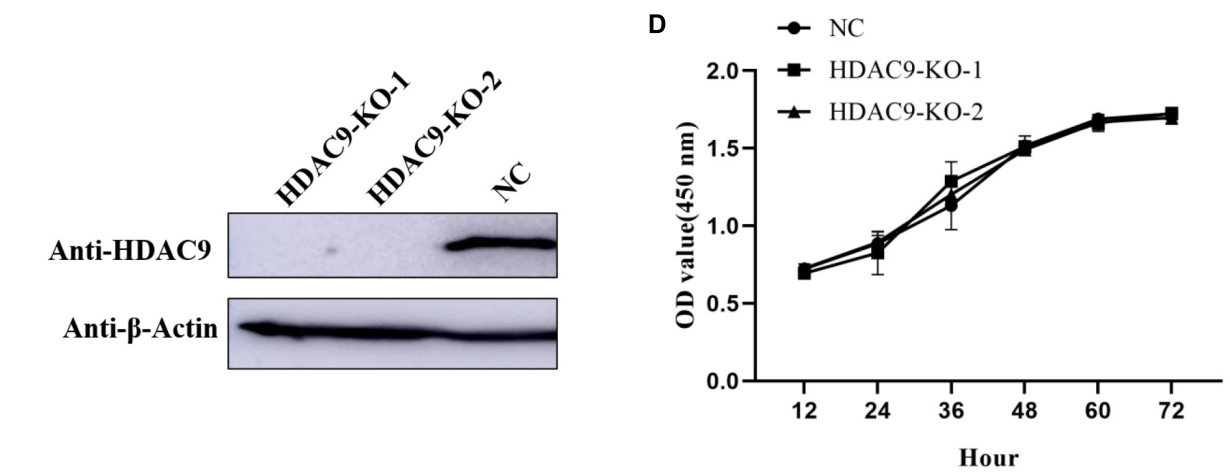

E

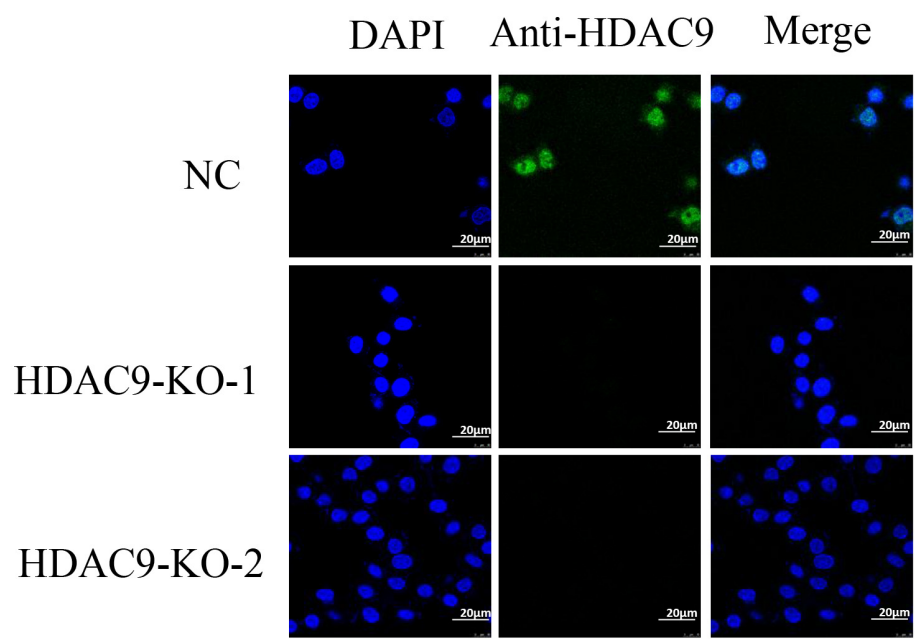

FIGURE 1 | Identification of the HDAC9 knockout BHK-21 cells. (A) Alignment of the NC (cells transfected with empty vector and selected by puromycin), HDAC9-KO-1, HDAC9-KO-2, and HDAC9 CDS sequences using DNAMAN software. The red box indicates the sgRNA sequence and the mutations in PAM motif. (B) Snapgene software was used to confirm the sequencing peaks of PCR products of NC, HDAC9-KO-1, and HDAC9-KO-2. (C) The expression of HDAC9 was detected by Western blotting with HDAC9 antibody, and $\beta$-Actin was used as a control to show the even loading of samples. (D) NC and HDAC9-KO cells (HDAC9-KO-1 and HDAC9-KO-2) were seeded in 96-well plates at $1 \times 10^{4}$ cells/well. CCK8 reagent was added to each well for $10 \mu \mathrm{L}$ and incubated for $1 \mathrm{~h}$, and then the absorption at $450 \mathrm{~nm}$ was determined. (E) Immunofluorescence assay of NC and HDAC9-KO cells (HDAC9-KO-1 and HDAC9-KO-2); the signals of HDAC9 protein were observed under a confocal microscope. Green signals represent HDAC9 and nucleus was stained with DAPI. Statistical significance was analyzed by Student's $t$-test: ${ }^{*} p<0.05,{ }^{* \star} p<0.01$. 
A

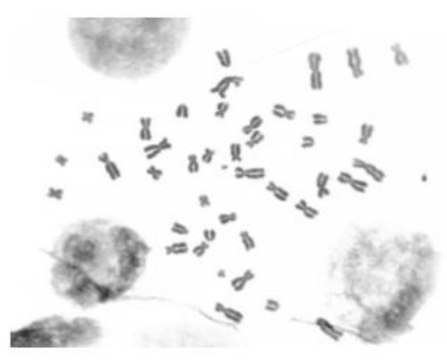

B

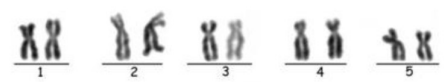

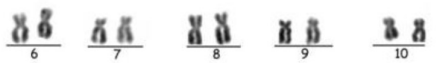

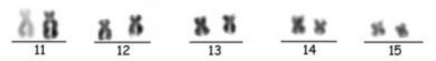

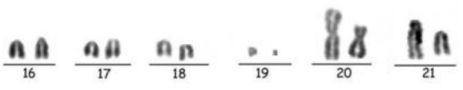

c

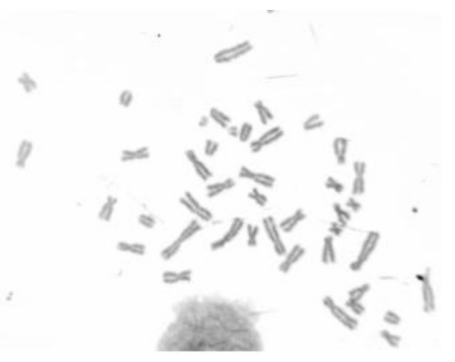

D

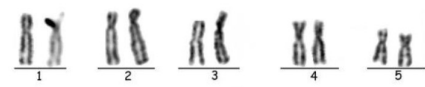

$\frac{\| \&}{6} \frac{A X}{7} \quad \frac{8 R}{8} \frac{M \&}{9} \frac{D D}{10}$

$\frac{X 8}{11} \quad \frac{8 \pi}{12} \quad \frac{x \wedge}{13} \quad \frac{8 x}{14} \frac{x \times}{15}$

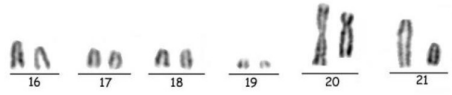

FIGURE 2 | Chromosomal analysis of NC and the HDAC9-KO-1 cell lines. (A) Chromosomes of NC with a diploid number of 42. (B) GTG-banded NC Karyotype. (C) Chromosomes of HDAC9-KO-1 with a diploid number of 42. (D) GTG-banded HDAC9-KO-1 karyotype.

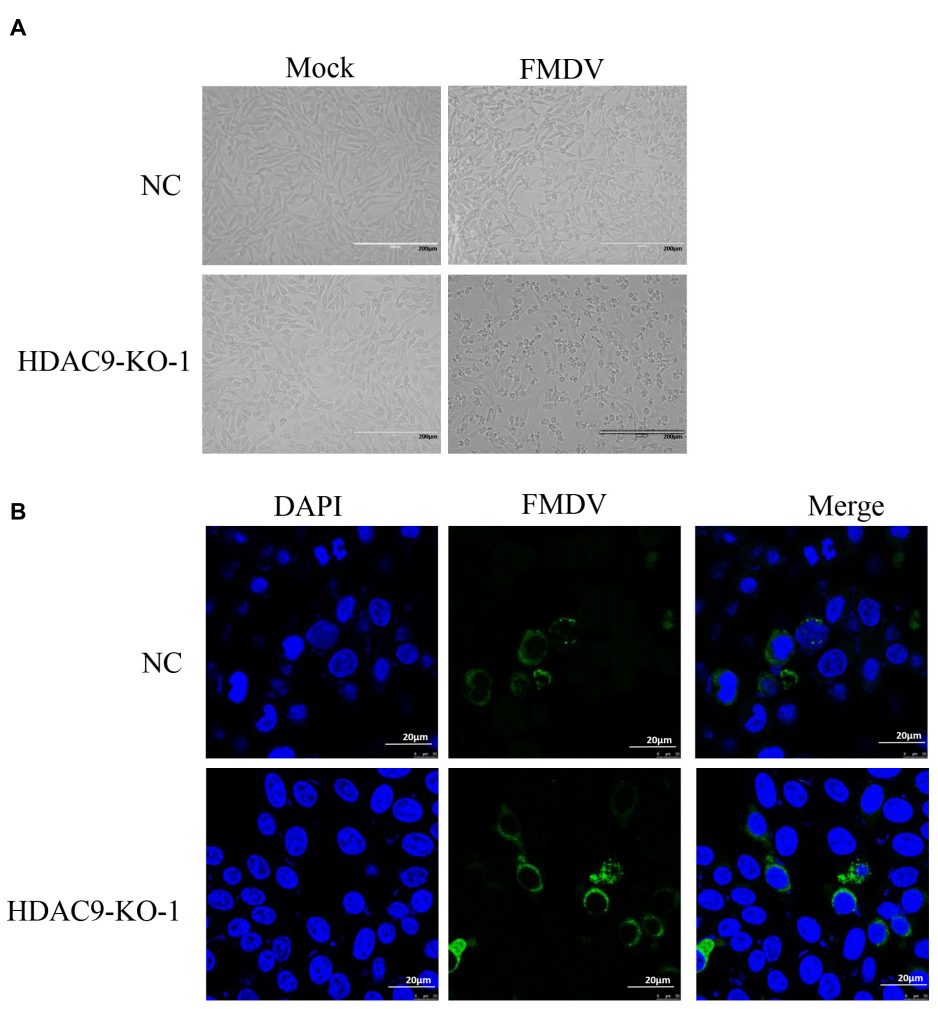

FIGURE 3 | Growth characteristics of FMDV. (A) CPE caused by FMDV, NC, and HDAC9-KO-1 cells was seeded in 6-well plates $\left(2 \times 10^{6}\right.$ cells/well) and were infected with FMDV at an MOI of 0.1 for $4 \mathrm{~h}$. (B) Immunofluorescence assay of NC and HDAC9-KO-1 cells infected with FMDV. The cells were infected with or without FMDV at an MOI of 0.1. After $6 \mathrm{~h}$, the signals of FMDV protein were observed under a confocal microscope. Green signals represent FMDV and nucleus was stained with DAPI. 
A

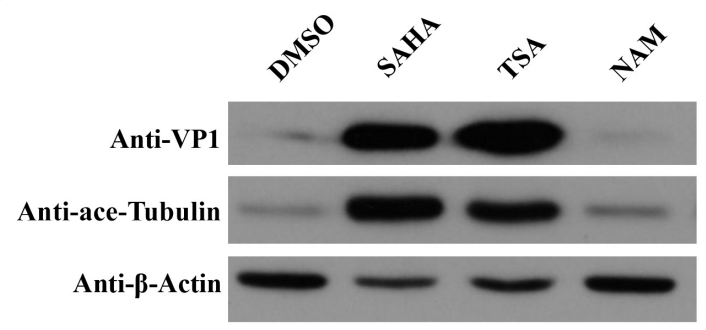

B
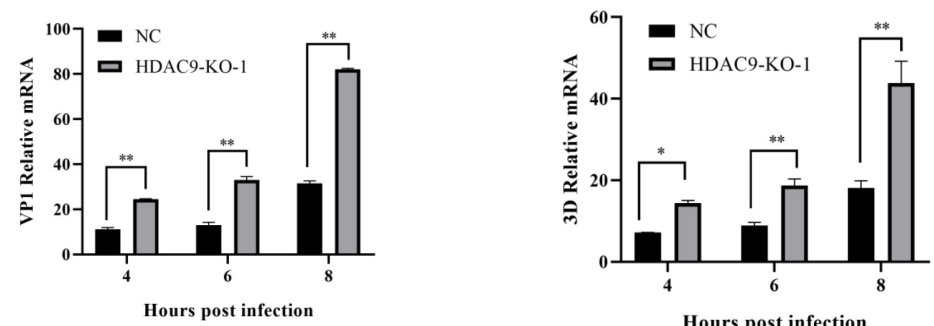

C

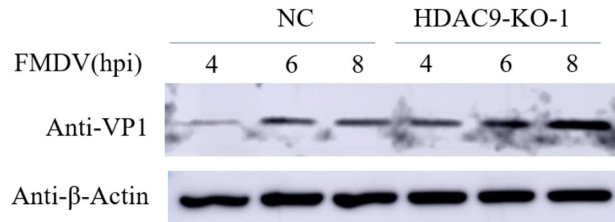

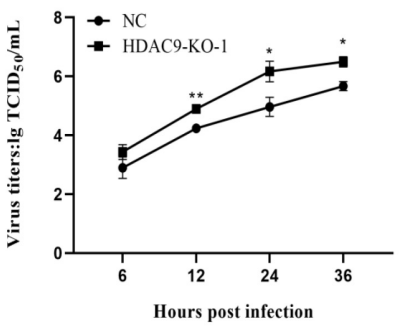

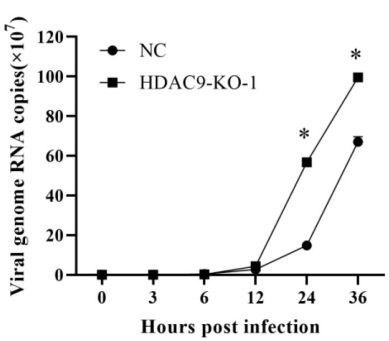

FIGURE 4 | HDAC9 knockout enhances FMDV replication. (A) BHK-21 cells were seeded in 60-mm dishes to reach an approximate $90 \%$ confluence and infected with FMDV at an $\mathrm{MOI}$ of 0.1 . Cells were incubated for $1 \mathrm{~h}$ and treated with HDAC inhibitors (TSA, SAHA, and NAM), respectively. Samples were collected at 6 hpi. VP1 protein and ace-tubulin were determined by Western blot with indicated antibodies. (B) HDAC9 knockout cells (HDAC9-KO-1) and NC were infected with equal amounts of FMDV at an $\mathrm{MOI}$ of 0.1 for 4,6 , and $8 \mathrm{~h}$. qRT-PCR was performed to examine the expression of FMDV VP1 and 3D relative mRNA. (C) The cells were infected with equal amounts of FMDV at the same time as in (B). The expression of FMDV VP1 protein was determined by Western blot, and $\beta$-Actin was used as an internal reference. (D) FMDV titers were determined by the TCID 50 method after cells were infected with FMDV at an MOI of 0.001. (E) NC and HDAC9-KO cells were separately seeded in 24-well plates $\left(5 \times 10^{5}\right.$ cells/well), and the cells were infected with virus at an $\mathrm{MOI}$ of 0.001 . Cells and supernatants were collected 0,3 , $6,12,24$, and 36 hpi, and the expression of viral RNA was determined using qRT-PCR. All experiments were repeated three times, with similar results. Statistical significance was analyzed by Student's $t$-test: ${ }^{*} p<0.05,{ }^{* *} p<0.01$.

total of 261 million raw sequencing reads and 259.75 million clean reads $(99.52 \%$ of total reads). To investigate the DEGs in NC and HDAC9-KO-1 cells after FMDV infection, an independent statistical hypothesis test was used to detect all DEGs, and the different test $p$-value was provided. Meanwhile, the DEGs were used to perform GO enrichment analysis.

As shown in Figures 6A,B, KEGG classification showed that the DEGs were mainly involved in signal transduction, immune system, endocrine system, transport and catabolism, cell growth, 


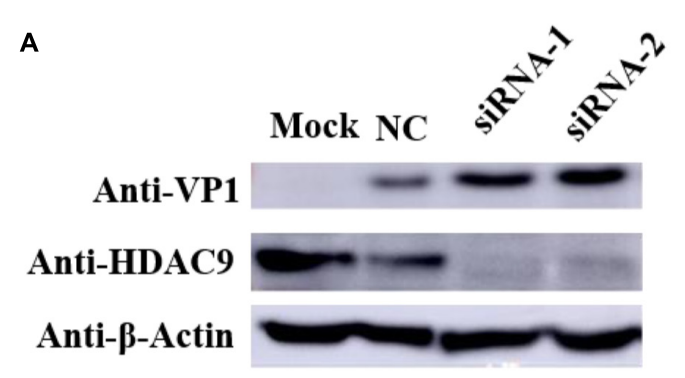

B

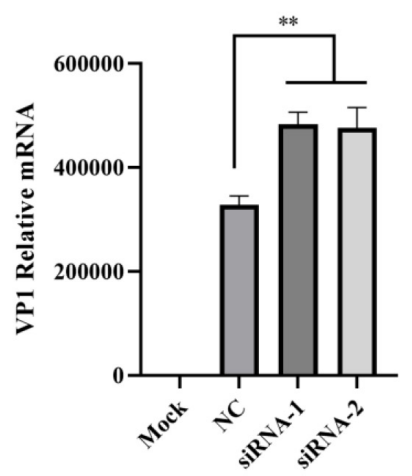

C

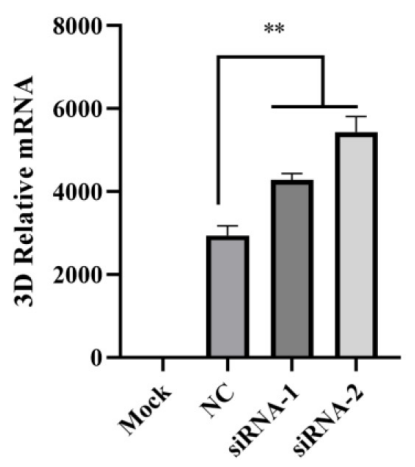

FIGURE 5 | Knockdown of HDAC9 enhances FMDV replication in PK-15 cells. PK-15 cells were transfected with control siRNA (NC) or siRNA targeting to HDAC9 for $24 \mathrm{~h}$, and then infected with FMDV $(\mathrm{MOI}=1)$ for $6 \mathrm{~h}$. (A) Western blot analysis was performed to detect the expression of HDAC9 and viral proteins VP1 with indicated antibodies. (B,C) Total RNA was extracted and qRT-PCR was performed to detect the expression of FMDV VP1 and 3D relative mRNA. All experiments were repeated three times, with similar results. Data are presented as mean $\pm \operatorname{SD}(n=3)$. Statistical significance was analyzed by Student's $t$-test: ${ }^{*} p<0.05$, ${ }^{* *} p<0.01$.

and death. The KEGG pathways were significantly enriched in the TNF, FoxO, p53, MAPK, IL-17, NF- $\mathrm{B}$, NOD-like receptor, Tolllike receptor, and RIG-I-like receptor signaling pathway. These pathways were mainly regulated to FMDV infection triggered anti-virus innate immune response.

The RT-qPCR analysis was used to detect the differential gene expression to verify the accuracy of the RNA-seq data. As shown in Figure 7, the immune response-related genes were randomly selected for analysis between FMDVinfected and uninfected NC and HDA9-KO-1 cells, including IGFBP5, CALM, IL2RG, BCL2L1, PLEKHA4, CXCL1/2/3, SOD2, CCL20, BIR3, NFKIBA, TRAF2, and CYLD. The results of RT-qPCR corresponded with RNA-seq data (Figure 6C). All results indicated that HDAC9 may inhibit FMDV replication by regulating genes in these innate immunity pathways.

\section{DISCUSSION}

A series of cell lines, including BHK-21, PK-15, IB-RS-2 (De Castro, 1970), and SK-6 (Kasza et al., 1972) have been established as an important tool of separation, culture and research for FMDV. Propagation of FMDV by cell culture is an important method to preserve viral biological and antigenic characteristics. Currently, the production of FMDinactivated vaccine is completely dependent on BHK-21 cells (Capozzo et al., 1997). BHK-21 cells were first established by MacPherson and Stoker using 1-day-old Syrian hamster kidney cells in 1962, which grow rapidly and have a wide sensitive spectrum of viruses, and were subsequently used for the proliferation of a variety of viruses and vaccine production (Regan and Petricciani, 1987), such as FMD disease vaccine, rabies vaccine, and Newcastle disease vaccine. However, there is still much room for improvement in viral susceptibility. The genome editing technology based on CRISPR-Cas9 has recently been applied widely to study gene function in many species. This technology has been used to modify genes that regulate the infection and immunity response of FMDV in BHK-21 cells and improve the replication efficiency of FMDV in BHK-21 cells, which will help to improve the yield or quality of FMD vaccine.

In this study, we established an HDAC9 knockout BHK-21 cell line by the CRISPR/Cas9 system. Compared with wild-type cells, these cells have a normal shape, size, and stable growth rate. Chromosome number and karyotype analysis showed that the HDAC9 knockout cell has 42 chromosomes like the wild-type BHK-21 cell and without chromosomal aberration.

HDAC9 belongs to class II HDACs, which shuttles between the nucleus and cytoplasm. In some cases, class II HDACs 
A

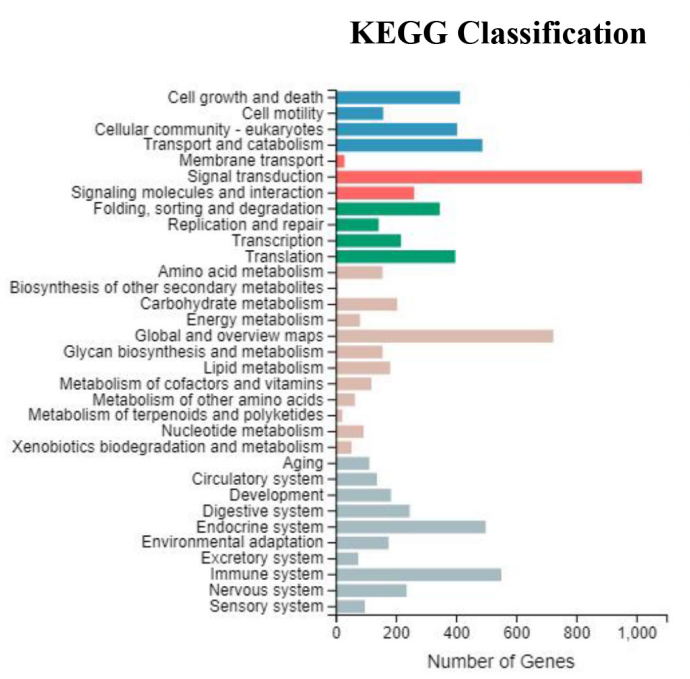

Cellular Processes

Environmental Information Processing

Genetic Information Processing

Metabolism

Organismal Systems
B

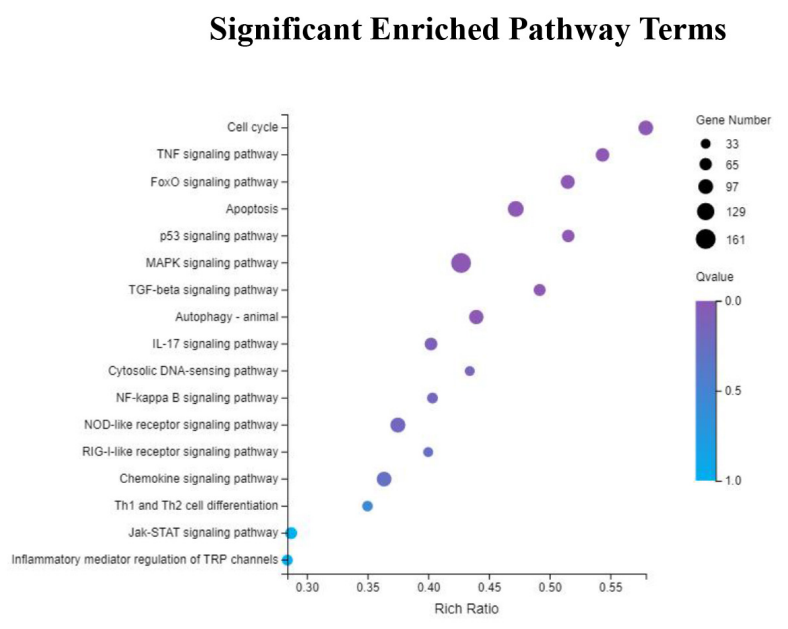

C

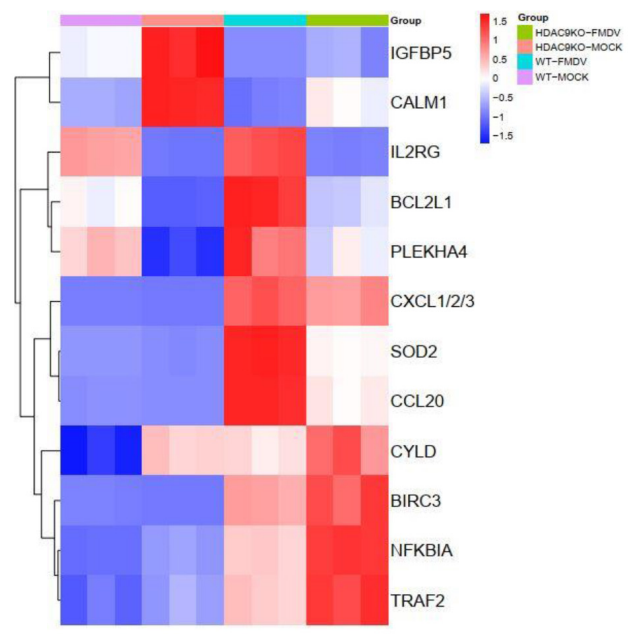

FIGURE 6 | KEGG classification and pathway enrichment analysis of DEGs. (A) KEGG classifications of DEGs. The $x$-axis indicates the number of DEGs and the $y$-axis indicates the different terms. (B) Significant enriched pathway results for the DEGs. The $x$-axis indicates the rich factor of GO and the $y$-axis indicates the GO term (the recommended cutoff of $p$-value is 0.05). (C) Heatmap of selected differentially expressed genes involved in immune response.

can also be used as transcriptional activators and results in inducible gene expression (Shakespear et al., 2011). So far, some studies have found that HDACs are the regulator of signal transduction activation in an innate immune response. HDAC9 directly maintains the deacetylation status of TBK1 and enhances its kinase activity to activate antiviral innate immunity (Li et al., 2016). We found that TRIM29 promoted DNA virus and RNA virus infections by inhibiting the innate immune response (Xing et al., 2017, 2018). TRIM29 is also called ataxia telangiectasia group D-complementing (ATDC); HDAC9 deacetylates the Lys ${ }^{116}$ of ATDC and changes the ability of ATDC to correlate with $\mathrm{p} 53$, thereby increasing the transcriptional activation function of p53 (Yuan et al., 2010). In addition, recent studies have shown that p53 inhibits innate immune response during FMD disease infection (Zhang et al., 2019). As a deacetylase, HDAC6 promotes RIG-I activation and innate antiviral immunity to recognize and restrict RNA virus infection (Liu et al., 2016). HDAC4 promotes type I interferon signaling and co-precipitate with STAT2 and restricts VACV and HSV-1 replication and spread (Lu et al., 2019). HDAC3 is an epigenetic regulator of the $\mathrm{CD} 8^{+}$T-cell cytotoxicity program, which inhibits $\mathrm{CD}^{+} \mathrm{T}$ cell cytotoxicity early during activation. HDAC3 was also found to modulate the signal transducer and activator of STAT3 to the LEAP-1 promoter. HDAC3 inhibitor treatment also blocked HCV replication in a mouse model of $\mathrm{HCV}$ infection (Zhou et al., 2018). 

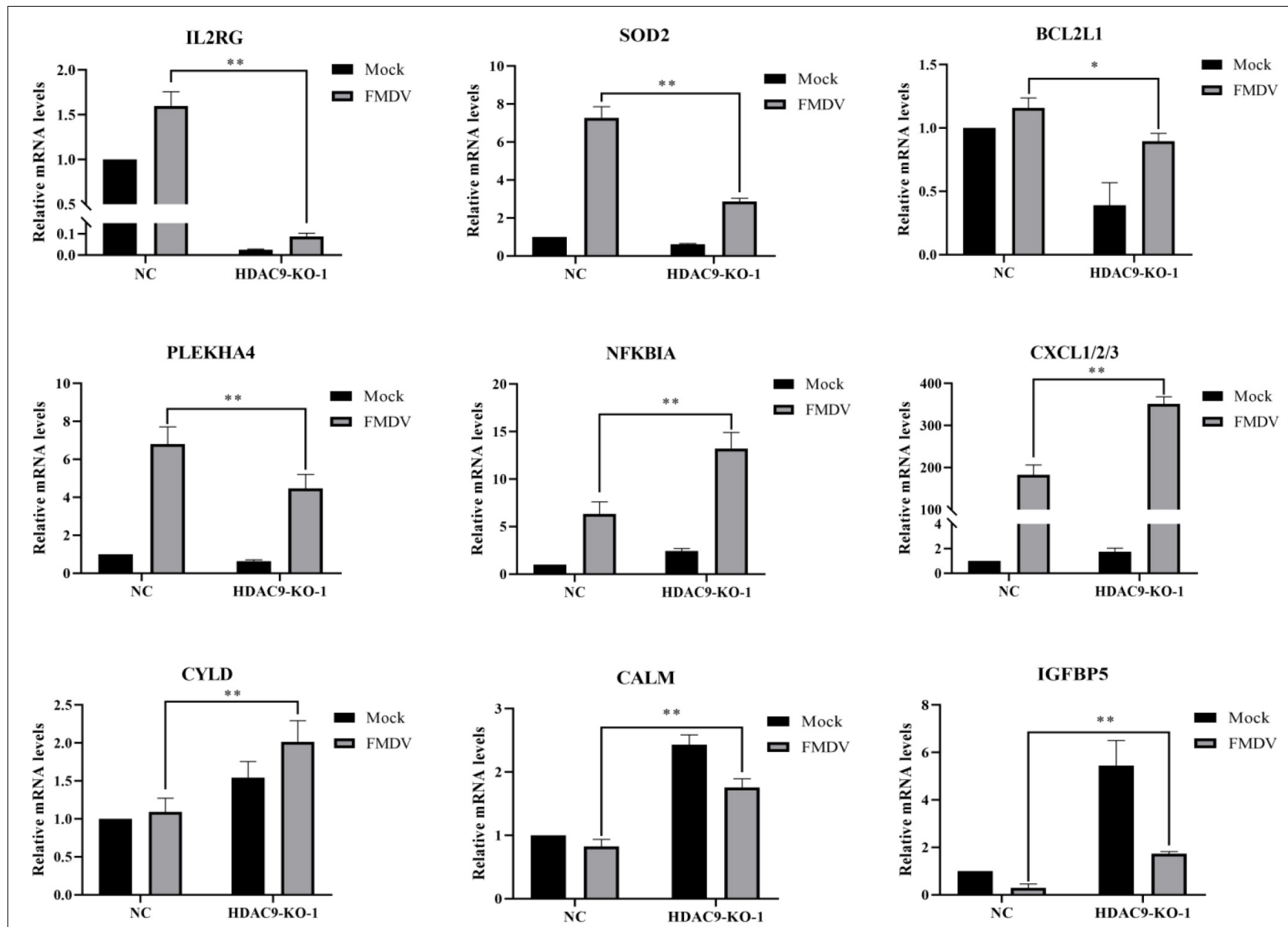

TRAF2
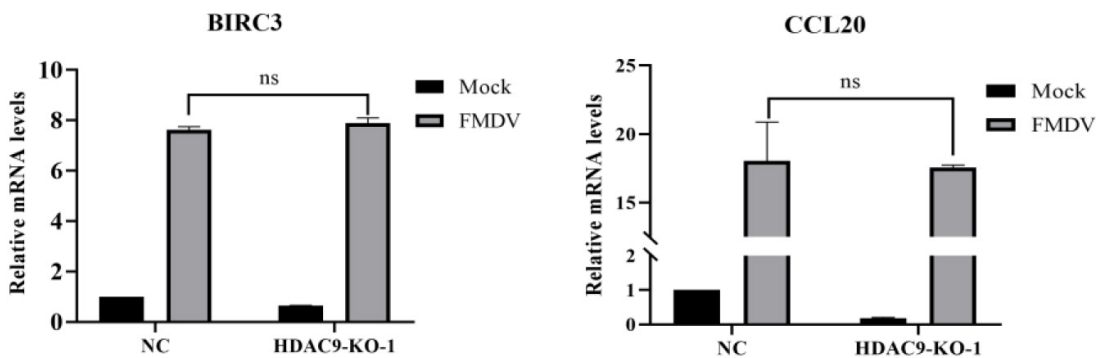

FIGURE 7 | Verification of RNA-seq data with RT-qPCR. Validation of the differentially expressed genes using the RT-qPCR assay in NC and HDAC9-KO-1 cells. Experiments were performed in triplicate and repeated three times with similar results. Data are presented as mean \pm SD $(n=3)$. Statistical significance was analyzed by Student's $t$-test: ${ }^{\star} p<0.05,{ }^{\star *} p<0.01$.

Our previous study found that FMDV replication was significantly higher in HDAC9 knockout cells than in NC cells. To explore the crucial role of HDAC9 in antiviral immune signaling pathways, RNA-seq was performed. We found that KEGG pathways were mainly enriched in the immune-related pathway, such as Jak-STAT, NOD-like receptor, Toll-like receptor, NF- $\mathrm{B}$, and MAPK signaling pathways. Then, multiple DEGs were selected for RT-qPCR detection. The results showed that the randomly selected genes validated by RT-qPCR displayed similar expression patterns with that in the RNA-seq data. Cytokines, including interleukin, interferons, tumor necrosis factor superfamily, colony-stimulating factor, chemokine, and growth factor, play an important role in preventing viral infection (Depalo, 2000). The transcription factor NF-кB plays a key role in coordinating the immune response. NF- $\kappa$ B inhibitor alpha (NFKBIA) inhibits NF- $\kappa \mathrm{B}$ by blocking the nuclear localization signal of NF- $\kappa B$ protein and keeps it inactive in the cytoplasm (Li and Verma, 2002). We found that compared with the NC 
cell line, the expression of NFKBIA in the HDAC9-KO cell line was significantly upregulated by FMDV infection. During FMDV infection, the cytokines CXCL1/2/3, IL2RG, SOD2 (superoxide dismutase 2), and PLEKHA4 were upregulated by the host to stimulate inflammatory response for virus clearance. However, the expressions of these genes were reduced in the HDAC9-KO cell line compared to NC cells after FMDV infection. On the contrary, IGFPB5 (insulin-like growth factorbinding protein 5) and CALM (calmodulin) were downregulated after FMDV infection. IGFBP5 has various IGF-independent cellular activities, including receptor-independent cellular uptake followed by transcriptional regulation (Goda et al., 2008). Recently, studies have shown that the p53 gene is related to the upregulated expression of IGFPB5 during cell inflammation (Fumihiro et al., 2018). The above genes mainly regulate signal transduction, innate antiviral immune response, cell growth, and death levels, and are enriched in Toll-like receptor signaling pathway, MAPK signaling pathway, NOD-like receptor signaling pathway, NF- $\kappa$ B signaling pathway, and p53 signaling pathway, which indicated that HDAC9 might inhibit FMDV infection by regulating genes in these pathways.

\section{CONCLUSION}

Protein acetylation and deacetylation play important roles in gene expression regulation. Acetylation or deacetylation is associated with the development of many diseases, such as cancer, neurological disorder, and inflammatory diseases. At the same time, acetylation also plays an important role in host antiviral innate immune response. In our preliminary study, HDAC9 can regulate the replication of FMDV. It is necessary to explore further the specific relationship between HDACs and FMDV, which will provide a more comprehensive understanding, theory, and application basis for the prevention and control of FMD.

\section{REFERENCES}

Alexandersen, S., and Mowat, N. (2005). Foot-and-mouth disease:host range and pathogenesis. Curr. Top. Microbiol. Immunol. 288, 9-42. doi: 10.1007/3-54027109-0_2

Amadori, M., Volpe, G., Defilippi, P., and Berneri, C. (1997). Phenotypic features of BHK-21 cells used for production of foot-and-mouth disease vaccine. Biologicals 25, 65-73. doi: 10.1006/biol.1996.0061

Capozzo, A. V. E., Periolo, O. H., Robiolo, B., Seki, C., Torre, J. L., and Grigera, P. R. (1997). Total and isotype humoral responses in cattle vaccinated with foot and mouth disease virus (FMDV) immunogen produced either in bovine tongue tissue or in BHK-21 cell suspension cultures. Vaccine 15, 624-630. doi: 10.1016/S0264-410X(96)00284-8

Choi, S. J., Lee, H. C., Kim, J. H., Park, S. Y., Kim, T. H., Lee, W. K., et al. (2016). HDAC6 regulates cellular viral RNA sensing by deacetylation of RIG-I. EMBO J. 35, 429-442. doi: 10.15252/embj.201592586

De Castro, M. P. (1970). Clonal variation in the swine kidney cell line, IB-RS-2, in relation to morphology, karyotype and susceptibility to the foot-and-mouth disease virus (FMDV). Arqs. Inst. Biol. 37, 103-127.

de Ruijter, A. J., van Gennip, A. H., Caron, H. N., Kemp, S., and van Kuilenburg, A. B. (2003). Histone deacetylases (HDACs):characterization of the classical HDAC family. Biochem. J. 370, 737-749. doi: 10.1042/BJ20021321

Depalo, O. V. A. (2000). Anti-Inflammatory Cytokines. Chest 117, 1162-1172. doi: $10.1378 /$ chest.118.2.503

\section{DATA AVAILABILITY STATEMENT}

The datasets presented in this study can be found in online repositories. The names of the repository/repositories and accession number(s) can be found below: https://www.ncbi.nlm. nih.gov/sra/PRJNA777823.

\section{AUTHOR CONTRIBUTIONS}

SH performed the experimental work, analyzed the data, and wrote the manuscript. SH and RM designed the experiments and assisted in manuscript preparation and revision. YS provided critical experimental design and modified the manuscript. All authors have read and agreed to the published version of the manuscript.

\section{FUNDING}

This study was supported by the grants from the Key Development and Research Foundation of Gansu (Grant Nos. 20YF3NA005 and 21YF5WA153) and the National Natural Science Foundation of China (No. 31772717).

\section{ACKNOWLEDGMENTS}

We thank Haixue Zheng (Lanzhou Veterinary Research Institute, Chinese Academy of Agricultural Sciences) for providing the anti-VP1 rabbit polyclonal antibody. Polyclonal pig antiserum against FMDV was provided by OIE/China National Foot-and-Mouth Disease Reference Laboratory (Lanzhou, Gansu, P.R. China).

Feng, Q., Miao, Y., Ge, J., Yuan, Y., Zuo, Y., Qian, L., et al. (2018). ATXN3 Positively Regulates Type I IFN Antiviral Response by Deubiquitinating and Stabilizing HDAC3. J. Immunol. 201, 675-687. doi: 10.4049/jimmunol.180 0285

Fumihiro, S., Yoshiaki, T., Jun, M., Rei, O., Hideo, S., Hiromi, R., et al. (2018). IGF Binding Protein-5 Induces Cell Senescence. Front. Endocrinol. 9:53. doi: $10.3389 /$ fendo.2018.00053

Glozak, M. A., Sengupta, N., Zhang, X., and Seto, E. (2005). Acetylation and deacetylation of non-histone proteins. Gene 363, 15-23. doi: 10.1016/j.gene. 2005.09.010

Goda, N., Tenno, T., Inomata, K., Shirakawa, M., Tanaka, T., and Hiroaki, H. (2008). Intracellular protein delivery activity of peptides derived from insulinlike growth factor binding proteins 3 and 5. Exp. Cell Res. 314, 2352-2361. doi: 10.1016/j.yexcr.2008.05.008

Grubman, M., and Baxt, B. (2004). Foot-and-mouth disease. Clin. Microbiol. Rev. 17, 465-493. doi: 10.1128/CMR.17.2.465

Haeussler, M., Sch Nig, K., Eckert, H., Eschstruth, A., Mianné, J., Renaud, J. B., et al. (2016). Evaluation of off-target and on-target scoring algorithms and integration into the guide RNA selection tool CRISPOR. Genome Biol. 17:148. doi: 10.1186/s13059-016-1012-2

Hatakeyama, D., Shoji, M., Yamayoshi, S., Yoh, R., Ohmi, N., Takenaka, S., et al. (2018). Influenza A virus nucleoprotein is acetylated by histone acetyltransferases PCAF and GCN5. J. Biol. Chem. 293, 7126-7138. doi: 10. 1074/jbc.RA117.001683 
Kasza, L., Shadduck, J. A., and Christofinis, G. J. (1972). Establishment, viral susceptibility and biological characteristics of a swine kidney cell line SK-6. Res. Vet. Sci. 13, 46-51. doi: 10.1016/S0034-5288(18)34087-6

Kiernan, R., Bres, V., Ng, R. W., Coudart, M. P., El Messaoudi, S., Sardet, C., et al. (2003). Post-activation turn-off of NF-kappa B-dependent transcription is regulated by acetylation of p65. J. Biol. Chem. 278, 2758-2766. doi: 10.1074/ jbc.M209572200

Klein, J. (2009). Understanding the molecular epidemiology of foot-and-mouthdisease virus. Infect. Genet. Evol. 9, 153-161. doi: 10.1016/j.meegid.2008. 11.005

Knowles, N. J., and Samuel, A. R. (2003). Molecular epidemiology of footand-mouth disease virus. Virus Res. 91, 65-80. doi: 10.1016/S0168-1702(02) 00260-5

Li, Q., and Verma, I. M. (2002). NF- $\kappa$ B regulation in the immune system. Nat. Rev. Immunol. 2, 725-734. doi: 10.1038/nri910

Li, X., Zhang, Q., Ding, Y., Liu, Y., Zhao, D., Zhao, K., et al. (2016). Methyltransferase Dnmt3a upregulates HDAC9 to deacetylate the kinase TBK1 for activation of antiviral innate immunity. Nat. Immunol. 17, 806-815. doi: 10.1038/ni.3464

Liu, H. M., Jiang, F., Loo, Y. M., Hsu, S., Hsiang, T. Y., Marcotrigiano, J., et al. (2016). Regulation of Retinoic Acid Inducible Gene-I (RIG-I) Activation by the Histone Deacetylase 6. EBioMed. 9, 195-206. doi: 10.1016/j.ebiom.2016.06.015

Lu, Y., Stuart, J. H., Talbot-Cooper, C., Agrawal-Singh, S., Huntly, B., Smid, A., et al. (2019). Histone deacetylase 4 promotes type I interferon signaling, restricts DNA viruses, and is degraded via vaccinia virus protein C6. Proc. Natl. Acad. Sci. U.S.A. 116, 11997-12006. doi: 10.1073/pnas.1816399116

Mao, R. Q., Sun, D. H., Yang, F., Tan, H., Zhu, Z. X., Zheng, H. X., et al. (2018). Establishment and Evaluation of a Stable Bovine Thyroid Cell Line for Investigating Foot-and-Mouth Disease Virus. Front. Microbiol. 9:2149. doi: $10.3389 /$ fmicb. 2018.02149

Nusinzon, I., and Horvath, C. M. (2003). Interferon-stimulated transcription and innate antiviral immunity require deacetylase activity and HDAC1. PNAS 100, 14742-14747. doi: 10.1073/pnas.2433987100

Ran, F. A., Hsu, P. D., Wright, J., Agarwala, V., Scott, D. A., and Zhang, F. (2013). Genome engineering using the CRISPR-Cas9 system. Nat. Prot. 8:2281. doi: 10.1038/nprot.2013.143

Reed, L. J., and Muench, H. (1937). A Simple Method of Estimating Fifty Per Cent Endpoints. Am. J. Trop.Med. Hygiene 27, 493-497.

Regan, P. J., and Petricciani, J. C. (1987). The approach used to establish the safety of veterinary vaccines produced in the BHK 21 cell line. Dev. Biol. Standardization 68:19.
Shakespear, M., Halili, M., Irvine, K., Fairlie, D., and Sweet, M. (2011). Histone deacetylases as regulators of inflammation and immunity. Trends. Immunol. 32, 335-343. doi: 10.1016/j.it.2011.04.001

Xing, J., Zhang, A., Minze, J. L., Li, X. C., and Zhang, Z. (2018). TRIM29 Negatively Regulates the Type I IFN Production in Response to RNA Virus. J. Immunol. 201, 183-192. doi: 10.4049/jimmunol.1701569

Xing, J., Zhang, A., Zhang, H., Wang, J., Li, X. C., Zeng, M. S., et al. (2017). TRIM29 promotes DNA virus infections by inhibiting innate immune response. Nat. Commun. 8:945. doi: 10.1038/s41467-01700101-w

Yuan, Z., Peng, L., Radhakrishnan, R., and Seto, E. (2010). Histone deacetylase 9 (HDAC9) regulates the functions of the ATDC (TRIM29) protein. J. Biol. Chem. 285, 39329-39338. doi: 10.1074/jbc.M110.179333

Zhang, T., Chen, H., Liu, X., Qi, L., Wu, R., and Gao, X. (2019). Basal Level p53 Suppresses Antiviral Immunity against Foot-and-Mouth Disease Virus. Viruses 11:727. doi: 10.3390/v11080727

Zhang, T. L., Chen, H. T., Qi, L. L., Zhang, J., Wu, R., Zhang, Y. G., et al. (2018). Transcript Profiling Identifies Early Response Genes against FMDV Infection in PK-15 Cells. Viruses 10:364. doi: 10.3390/v10070364

Zhou, Y., Wang, Q., Yang, Q., Tang, J., Xu, C., Gai, D., et al. (2018). Histone Deacetylase 3 Inhibitor Suppresses Hepatitis C Virus Replication by Regulating Apo-A1 and LEAP-1 Expression. Virol. Sin. 33, 418-428. doi: 10.1007/s12250018-0057-7

Conflict of Interest: The authors declare that the research was conducted in the absence of any commercial or financial relationships that could be construed as a potential conflict of interest.

Publisher's Note: All claims expressed in this article are solely those of the authors and do not necessarily represent those of their affiliated organizations, or those of the publisher, the editors and the reviewers. Any product that may be evaluated in this article, or claim that may be made by its manufacturer, is not guaranteed or endorsed by the publisher.

Copyright (c) 2022 Hou, Wang, Ren, Meng, Yin, Zhang, Tarasiuk, Pejsak, Jiang, Mao, Zhang and Sun. This is an open-access article distributed under the terms of the Creative Commons Attribution License (CC BY). The use, distribution or reproduction in other forums is permitted, provided the original author(s) and the copyright owner(s) are credited and that the original publication in this journal is cited, in accordance with accepted academic practice. No use, distribution or reproduction is permitted which does not comply with these terms. 